\title{
3D Reconstruction Human Body From Anthropometric Measurements Using Diversity Control Oriented Genetic Algorithm
}

\author{
Hoang Ngoc Thach, Nguyen Tien Dat ${ }^{\bowtie}$ \\ Modeling and Simulation Center, Viettel High Technology Industries Corporation, Vietnam \\ thachhn3@viettel.com.vn, datnt65@viettel.com.vn ${ }^{\bowtie}$
}

\begin{abstract}
3D digitalization of the human body has been studied extensively for various applications in anthropology, ergonomics, healthcare, entertainment and fashion industries. There are different methods and approaches to reconstruct the 3D body model namely using RGB cameras, depth cameras, scanning systems or anthropometric measurements of the human body. Generally, most of existing approaches have to tackle issues relating to security of personal data, the impact of the surrounding environment, cost of 3D scanning systems and complication of anthropometric measurements. This study proposes a method using simple body measurements and given body shapes to digitalize the human body. The effectiveness of proposed method is evaluated and demonstrated based on two datasets: a synthetic dataset generated from a parametric model and a real dataset on Vietnamese collected by Viettel Military Industry and Telecoms Group (Vietnam).
\end{abstract}

Keywords: 3D Reconstruction, anthropometric measurements, DCGA.

Received: 15 June 2021 Accepted: 19 June 2021 Published: 21 June 2021

\section{Introduction}

3D human body reconstruction has been widely applied to a broad range of areas such as health monitoring, virtual clothing try-on, e-commerce and security. For years, developing techniques for reshaping the 3D human body from anthropometric parameters has attracted a lot of research attention [6]. Yanhong Zeng in [9] proposed a method using a feature-selection-based local mapping for anthropometric modeling. Their system used limited anthropometric parameters as input to generate the $3 \mathrm{D}$ shapes and create a tool to show the advantage of the system. Ref [8] predicted face and body shape using non-linear optimization. Firstly, the shape of the model was optimized concerning the learned shape space that finds the best suitable points. Secondly, a mesh-based optimization was performed to tackle shape variations staying outside training data. Sergi Pujades [4] contributed notably to the field by building an application for quick generating 3D body shapes using measurements based on linear regressors. The study, (1) introduced a tool called The Virtual Caliper empowering users to rapidly measure their own bodies and create a metrically precise avatar; (2) provided a desktop application for research community to create $3 \mathrm{D}$ avatars which could be immediately animated. Ref [7] proposed another approach which created 3D mesh based on generic adjectives, height and weight to build human body shape based on only a few descriptions of users relating to "crowdshaped" bodies.

The main contributions of this paper are: (1) Proposing a state-of-the-art algorithm for 3D reconstruction of human model from body measurements, (2) Evaluating and validating research results based on both synthetic data and real data. The paper is organized as follows: Section II describes our approach on estimating 3D shape model from anthropometric measurements, including introduction of research methodology and demonstration of algorithms and techniques applied. Results evaluation and analysis are given in Section III, followed by the future work and conclusion in Section IV.

\section{Methodology}

This part is divided into 3 main steps: (1) Briefing the parametric model used, (2) Interpreting Diversity Control Oriented Genetic Algorithm (DCGA) and (3) Describing the implementation of the research.

\subsection{The SMPL Model}

In this research, a parametric model - The Skinned Multi-Person Linear (SMPL) model is used as a base to create an approximation of human body. The SMPL model [3] could be transformed from shape to shape based on 10 control shape parameters and 72 control pose parameters. SMPL was learned and created from CAESAR which is the largest commercially available scan database until now. Body mesh of SMPL model is pre-defined topology with 6890 vertices and type of surface mesh is triangle. The vertices $v \in R^{3}$ are modeled linearly by $\beta$ as follows:

$$
v=M \beta+\mu
$$




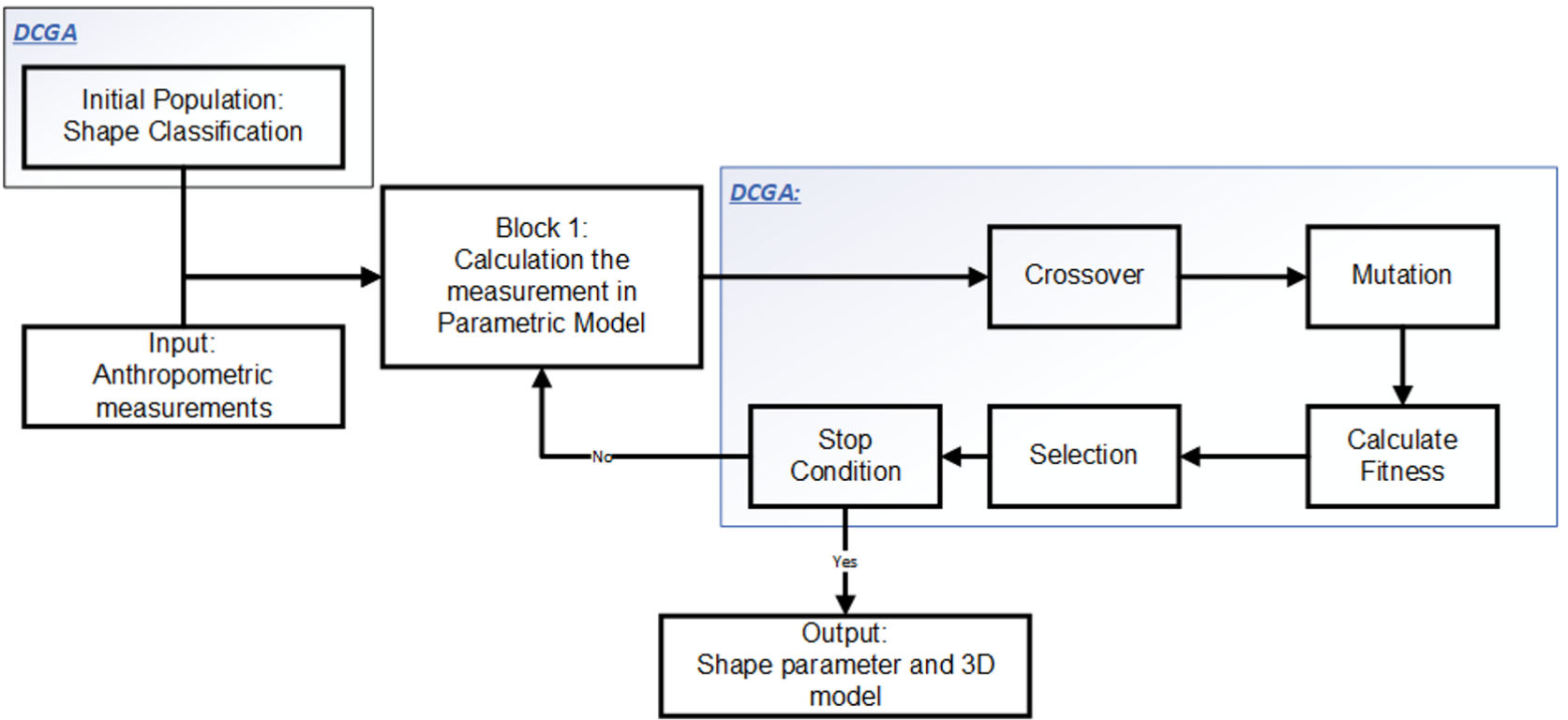

Figure 1: The proposed system for 3D Shape Reconstruction from Anthropometric Measurements.

\subsection{Diversity Control Oriented Genetic Algorithm (DCGA)}

In this section we present a system using Diversity Control Oriented Genetic Algorithm (DCGA) [5] which is a variant of the Genetic Algorithm (GA). Before digging deeper into DCGA, a summary of Heuristic optimization algorithm is given. It is a computational $\beta$ procedure which finds out an optimal solution by iteratively continuing to improve a candidate solution. GA is a class of Heuristic optimization method which mimics the evolutionary processes in nature such as reproduction or natural selection. Individual in the GA represent a solution for the problem with chromosomes represented by a vector. Generally, two vital steps including natural selection and reproduction are used repeatedly in each iteration (generation), in which reproduction is divided into hybridization and mutation processes. Before entering the evolutionary process in each generation, a set of individuals (population) is initialized by using uniform random numbers.

Fig. 1 illustrates an overview of our system. We create a process of optimization to take DCGA as input anthropometric measurements and generate parameters as output for SMPL. Firstly, a set of shape parameters $(\beta)$ which is taken from Initial Population is applied to generate a 3D model based on SMPL. Block 1 is used to calculate the measurements of the parametric model which are identical to the inputs. In this case, each set of shape parameters is called a chromosome and each variable beta is gene. The solution space is defined as $\mathrm{V}$ for finding an approximate solution.

$$
\begin{aligned}
& \text { Chromosome : } \beta=\left[x_{1}, x_{2}, \ldots, x_{10}\right] \in V \\
& \text { Gene : } x_{i} \in \beta
\end{aligned}
$$

\subsubsection{Initial Population}

A general problem of traditional genetic algorithms is convergence to a local optimum, mainly because population structure is not diverse enough and too alike. We propose using the K-means clustering algorithm to initialize the population. A large data set of 50000 sets of $\beta_{j} \in V^{10}$ is randomly generated then $\mathrm{K}$-means is used to reinitializing the dataset into $\mathrm{N}$ clusters. The central component of each cluster will be the chromosomes of each individual in the initial population.

\subsubsection{Selection}

The selection is the process of selecting individuals from the initial population to be parents which could mate and recombine to create off-springs for the next generation. One of the main tasks in GA is ensuring the diversity in the population. DCGA is proposed for natural selection to improve population diversity after each generation. It includes 2 main steps: (i) Eliminating the duplicate structure/individual, (ii) Using Cross-generational Probabilistic Survival Selection (CPSS) method to select structure/individual. Evaluation function is used to arrange the order of individual. Evaluation function $(\mathrm{F})$ is created based on the loss function between $y$ - the input parameter of measurements and $\hat{y}$ - the estimated parameter of measurements which is defined from shape parameters $(\beta$ - individual).

$$
\begin{gathered}
L(y, \hat{y})=\sqrt{\sum k\left(y_{i}-\widehat{y}_{i}\right)^{2}} \\
F(\beta)=\frac{1}{L(y, \hat{y})}
\end{gathered}
$$




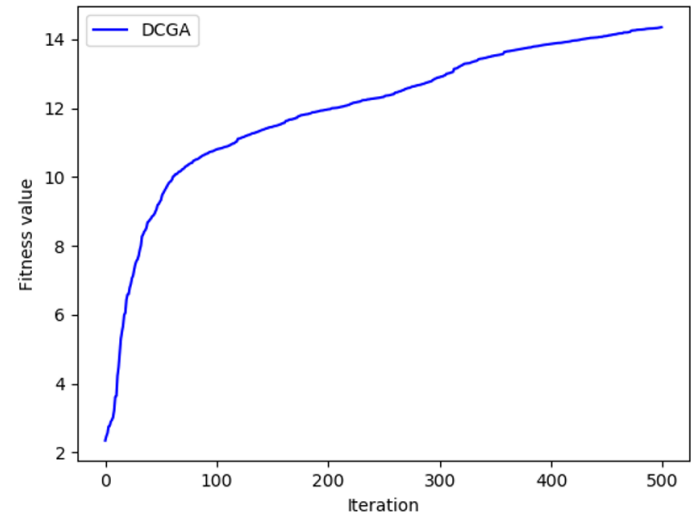

(a)

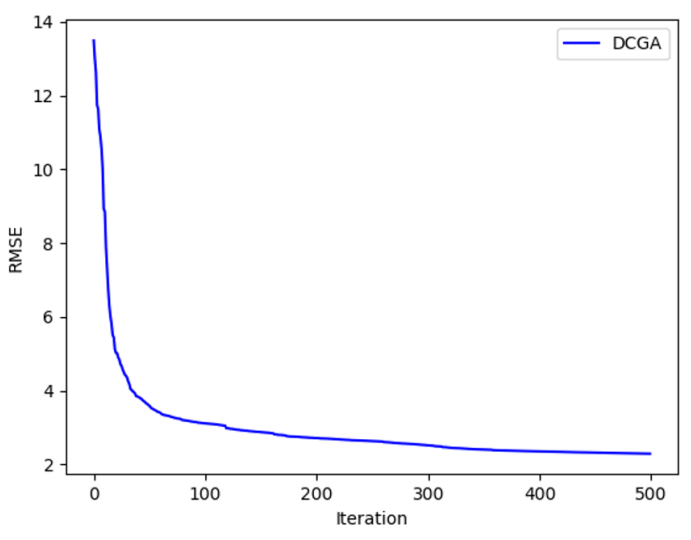

(b)

Figure 2: (a) Fitness value vs Generation number, (b) Root Mean Square Error vs Generation number.

Where $k$ is the coefficient to ensure the value of evaluation function is not too huge or reach zero. After arranging, the first individual is chosen and the next is selected based on the basis of a probability defined by the following equation:

$$
p=\left[(1-c) \frac{h}{M}+c\right]^{\alpha}
$$

Where $h$ is the hamming distance between a candidate individual and the individual which have corresponding genes satisfied: $\left|x_{i}^{1}-x_{i}^{2}\right|>\varepsilon, 0<\varepsilon \ll 1 ; M$ is the number of genes in an individual; $c$ and $\alpha$ are the coefficients for shape and exponent whose value are in the range of $[0,1]$. (iii) If the number of individuals after step (ii) is smaller than $\mathrm{N}$, new individuals will be generated randomly in the initial population.

\subsection{Reproduction}

The fittest individuals are selected and pass their genes to the next generation. Two common phases in reproduction are crossover and mutation. There are different methods and algorithms to implement the crossover and mutation such as ref [1] and [2]. In this research, we use Laplace crossover and exponential mutation to ensure the effectiveness for real numbers.

\section{1) Crossover}

Laplace crossover (LX) for individuals:

$\beta^{1}=\left(\beta_{1}^{1}, \beta_{2}^{1}, \ldots, \beta_{10}^{1}\right)$ and $\beta^{2}=\left(\beta_{1}^{2}, \beta_{2}^{2}, \ldots, \beta_{10}^{2}\right)$ are presented as follows: (i) Generating a random number $u \in[0,1]$ following a uniform distribution, (ii) a random number $\mathrm{v}$ is generated based on the Laplace distribution:

$$
v= \begin{cases}a-b \ln (u), & u \leq \frac{1}{2} \\ a+b \ln (u), & u>\frac{1}{2}\end{cases}
$$

Where $a \in R$ is the position parameter, $b>0$ is scale parameter, if $b$ is small, the probability to generate the individuals which are similar to their parents is higher and vice versa. The two of individual are given as below:

$$
\begin{aligned}
& \beta_{i}^{\text {new }}=\beta_{i}^{1}+v\left|\beta_{i}^{1}-\beta_{i}^{2}\right| \\
& \beta_{i}^{\text {new }}=\beta_{i}^{2}+v\left|\beta_{i}^{1}-\beta_{i}^{2}\right|
\end{aligned}
$$

\section{2) Mutation}

Some of genes was used to a mutation with a random probability $p_{m}$. This research uses power mutation (PM) with genes $\beta=\left(\beta_{1}, \beta_{2}, \ldots, \beta_{10}\right)$. A random number $r \in[0,1]$ is generated following a uniform distribution and $\mathrm{s}$ is generated based on the power distribution $\left(x^{p}\right)$. New individuals are created by the following equation:

$$
\beta_{i}^{\text {new }}= \begin{cases}\beta_{i}-s\left(\beta_{i}-l_{i}\right), & t<r \\ \beta_{i}+s\left(r_{i}-\beta_{i}\right), & t \geq r\end{cases}
$$

where $t=\frac{\beta_{i}-l_{i}}{r_{i}-l_{i}}, l_{i}$ and $r_{i}$ are upper and lower bounds of a decision variable.

\subsection{Implementation Details}

The main code is implemented in Python and a popular library: NumPy. According to above analyses and the given problem, the value of each process is represented as follows: population size $N=50$, mutation probability $p_{m}=10$, exponent for probability function $\alpha=0.21$, shape coefficient $c=0.01$, scale parameter $b=1$, the index of the power distribution in mutation $p=0.75$. 
Table 1: Body shape classification.

\begin{tabular}{|c|c|c|}
\hline Body type & Description & Illustration \\
\hline $\begin{array}{l}\text { Hourglass } \\
(\mathrm{HG})\end{array}$ & $\begin{array}{l}\text { - Bust and hip are nearly equal in size } \\
\text { - Small and defined waist that is narrower than shoulder, bust and hip } \\
\text { - Slightly round shoulder } \\
\text { - Upper body is proportionate in length to well-shaped legs }\end{array}$ & \\
\hline $\begin{array}{l}\text { Triangle } \\
(\mathrm{T})\end{array}$ & $\begin{array}{l}\text { - Shoulder is clearly narrower than hip } \\
\text { - A defined waist thanks to larger hip }\end{array}$ & \\
\hline Oval $(\mathrm{O})$ & $\begin{array}{l}\text { - Shoulder and hip are both narrow and often proportional } \\
\text { - Bust is large } \\
\text { - Waist is undefined and is the widest part of the body }\end{array}$ & \\
\hline $\begin{array}{l}\text { Rectangle } \\
(\mathrm{R})\end{array}$ & $\begin{array}{l}\text { - Shoulder, bust and hip are around the same width } \\
\text { - Undefined waist } \\
\text { - Lack of curves throughout the body frame }\end{array}$ & \\
\hline $\begin{array}{l}\text { Inverted } \\
\text { Triangle } \\
\text { (IT) }\end{array}$ & $\begin{array}{l}\text { - Shoulders are noticeably wider than the hip-line } \\
\text { - Hip is small } \\
\text { - Little to no waist definition }\end{array}$ & \\
\hline
\end{tabular}

\section{Results and Discussion}

Research results are validated by 2 datasets: (1) synthetic dataset which was generated randomly from SMPL, (2) real dataset of Northern Vietnamese which was collected by Viettel Military Industry and Telecoms Group (Vietnam). The evaluation is implemented by both quantitative and qualitative analyses. To verify the quantitative results using anthropometric measurements and ensure the fairness and accuracy of proposed algorithm, we take into account 2 factors: difference between the input values and measurements of model and the errors between the measurements that are not used as input values.

\subsection{Synthetic Dataset on SMPL}

Based on the data clusters defined above, synthetic data is classified into 5 main groups according to different proportions between measurements to ensure the diversity of distribution in body shape. These datasets are used to validate and verify the efficient of DCGA. 05 groups of people are: (1) Hourglass, (2) Triangle, (3) Oval, (4) Rectangle, (5) Inverted Triangle, arranged in order as Table 1. Each group of people includes 50 samples according to the uniform distribution and the constrained conditions to ensure the correction subgroups.

\subsubsection{Quantitative Results}

Table 2 illustrates the errors between the values which set as inputs for DCGA algorithm and the equivalent values in generated model. Overall, the mean values for five types of people are lower than $4 \mathrm{~mm}$ and the best performance could be seen in the hip circumference. The results in triangle type are generally higher than the other body types, approximately $2.3 \mathrm{~mm}$. In term of input measurements, the arm length errors are given bigger than others. Based on the information as seen in table 3 , the triangle shape provides the most stable results.

The range from upper bound to lower bound is approximately $9 \mathrm{~mm}$, in contrast to nearly $40 \mathrm{~mm}$ of inverted triangle shape. The numbers of hourglass, rectangle and oval types are $14 \mathrm{~mm}, 14.5 \mathrm{~mm}$ and $17 \mathrm{~mm}$ respectively. However, the distributions of oval type have more outliers than others. Four over ten measurement errors in oval type including (1) Waist Circumference, (2) Shoulder to crotch, (3) Hip circumference, (4) Inside leg length have a greater number of outliers. On the other hand, most of the measurements in triangle shape has the smallest outside value. Rectangle and inverted triangle types have stayed in the middle range with only few abnormal values.

Table 4 illustrates errors from five body shape types in various measurements. The information shows mean error between twelve indexes in millimetres (lower than seven millimetres). Overall, the error in thigh circumference and total crotch length are the highest value, whereas upper arm indicates the lowest value throughout the whole measurements. The other measurements maintain the error in the range of approximately [2.5 $\mathrm{mm}, 8.5 \mathrm{~mm}]$. In thigh circumference, the mean error of Rectangle and Inverted Triangle shape are $14.63 \mathrm{~mm}$ and $12.79 \mathrm{~mm}$ respectively. For others, this circumference decreases slightly but keeps around $11 \mathrm{~mm}$. Similarly, the crotch length gives the lowest error in oval shape and is approximately $9.5 \mathrm{~mm}$, but slightly increases to the other shapes. On the other hand, the mean errors of hourglass and triangle are the smallest and around $1.5 \mathrm{~mm}$ as compared to other shape types.

Table 5 indicates the distribution of the whole errors in measurements. Generally, the inverted triangle shape has the largest range of data scale, whereas the smallest range is belonging to the oval shape. The range from upper bound to lower bound of oval type 
Table 2: The errors between the Input measurements and the results of model. Units are in $\mathrm{mm}$ for all values.

\begin{tabular}{|l|l|l|l|l|l|}
\hline & \multicolumn{1}{|c|}{ HG } & \multicolumn{1}{c|}{ T } & \multicolumn{1}{c|}{ O } & \multicolumn{1}{c|}{$\mathbf{R}$} & \multicolumn{1}{c|}{ IT } \\
\hline Overall Height & $1.95 \pm 1.45$ & $2.10 \pm 1.88$ & $2.87 \pm 2.44$ & $2.89 \pm 2.69$ & $2.00 \pm 1.90$ \\
\hline Shoulder breadth & $2.92 \pm 3.53$ & $3.53 \pm 2.40$ & $5.12 \pm 4.08$ & $4.05 \pm 3.77$ & $3.35 \pm 3.00$ \\
\hline Shoulder to crotch & $2.92 \pm 2.55$ & $2.18 \pm 1.72$ & $4.48 \pm 5.36$ & $4.43 \pm 3.71$ & $2.62 \pm 2.33$ \\
\hline Arm length & $4.81 \pm 4.38$ & $2.92 \pm 2.68$ & $5.49 \pm 4.20$ & $5.13 \pm 3.92$ & $5.58 \pm 5.43$ \\
\hline Chest circumference & $1.89 \pm 2.16$ & $1.82 \pm 1.54$ & $2.80 \pm 2.69$ & $3.02 \pm 2.42$ & $2.15 \pm 1.94$ \\
\hline Bust point to bust point & $3.62 \pm 2.60$ & $2.96 \pm 2.33$ & $3.97 \pm 3.25$ & $3.56 \pm 2.85$ & $3.61 \pm 2.24$ \\
\hline Waist circumference & $1.51 \pm 1.35$ & $2.22 \pm 1.61$ & $4.01 \pm 5.05$ & $4.32 \pm 3.34$ & $2.74 \pm 2.19$ \\
\hline Upper Hip Circumference & $1.89 \pm 1.77$ & $2.18 \pm 1.51$ & $2.76 \pm 2.27$ & $2.81 \pm 2.15$ & $1.98 \pm 1.67$ \\
\hline Hip circumference & $0.99 \pm 0.85$ & $1.09 \pm 0.91$ & $2.52 \pm 2.70$ & $2.12 \pm 2.09$ & $1.34 \pm 1.29$ \\
\hline Inside leg length & $3.65 \pm 3.27$ & $2.98 \pm 2.50$ & $3.83 \pm 6.72$ & $3.38 \pm 4.25$ & $2.72 \pm 3.00$ \\
\hline Mean & 2.61452 & 2.3981 & 3.7861 & 3.56954 & 2.81034 \\
\hline
\end{tabular}

Table 3: The distribution of errors between input measurements and results.

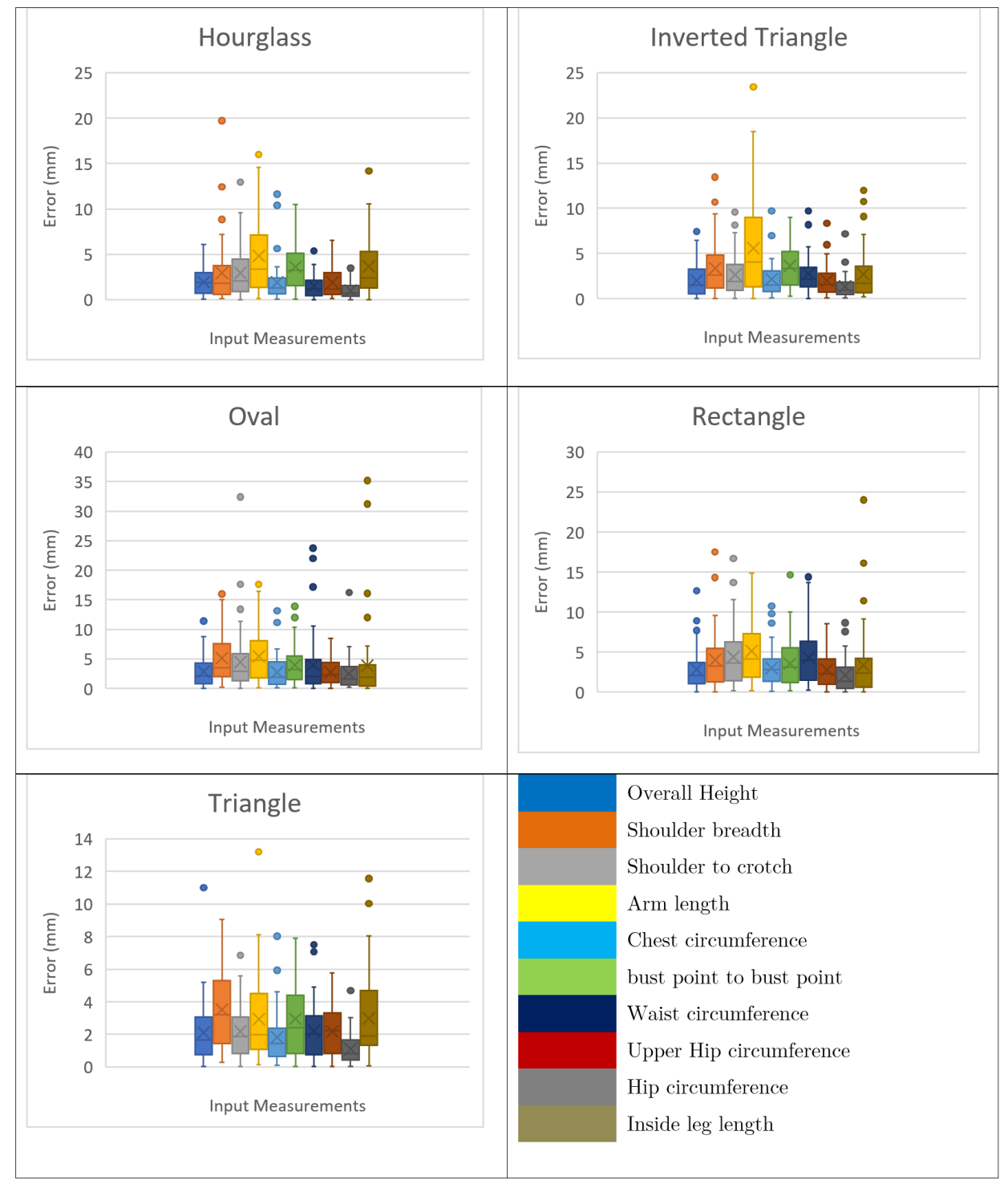


Table 4: The errors between the other measurements and the result of model. Units are in mm for all values.

\begin{tabular}{|l|l|l|l|l|l|}
\hline & \multicolumn{1}{|c|}{ HG } & \multicolumn{1}{|c|}{ T } & \multicolumn{1}{|c}{ O } & \multicolumn{1}{c|}{ R } & \multicolumn{1}{|c|}{ IT } \\
\hline Head circumference & $3.25 \pm 2.60$ & $3.96 \pm 2.64$ & $4.58 \pm 3.94$ & $4.24 \pm 3.68$ & $3.52 \pm 2.56$ \\
\hline Neck circumference & $4.88 \pm 4.31$ & $5.64 \pm 3.92$ & $5.32 \pm 4.74$ & $6.79 \pm 5.21$ & $6.43 \pm 5.16$ \\
\hline Bicep circumference & $6.74 \pm 5.35$ & $6.48 \pm 4.67$ & $6.87 \pm 5.05$ & $8.60 \pm 6.51$ & $7.43 \pm 6.16$ \\
\hline Thigh circumference & $12.61 \pm 9.51$ & $11.16 \pm 7.29$ & $10.45 \pm 8.53$ & $14.63 \pm 9.81$ & $12.79 \pm 9.89$ \\
\hline Chest width & $3.64 \pm 4.52$ & $3.63 \pm 3.73$ & $5.92 \pm 5.27$ & $5.08 \pm 4.11$ & $4.20 \pm 5.03$ \\
\hline Across back width & $3.10 \pm 2.71$ & $3.72 \pm 2.50$ & $3.65 \pm 2.50$ & $4.24 \pm 2.61$ & $3.59 \pm 2.44$ \\
\hline Upper arm length & $1.55 \pm 1.34$ & $1.55 \pm 1.26$ & $1.84 \pm 1.58$ & $2.18 \pm 1.69$ & $2.28 \pm 1.47$ \\
\hline Under bust girth & $6.39 \pm 5.00$ & $6.54 \pm 4.76$ & $6.19 \pm 4.63$ & $6.55 \pm 5.12$ & $7.16 \pm 4.74$ \\
\hline Back neck point to waist & $2.62 \pm 1.88$ & $2.78 \pm 2.06$ & $3.94 \pm 3.87$ & $3.72 \pm 2.63$ & $3.13 \pm 2.32$ \\
\hline Side neck point to waist & $4.66 \pm 4.10$ & $5.16 \pm 3.26$ & $5.82 \pm 4.98$ & $6.47 \pm 5.08$ & $5.74 \pm 4.47$ \\
\hline Knee height & $2.36 \pm 1.92$ & $2.54 \pm 2.04$ & $4.05 \pm 3.75$ & $3.72 \pm 3.52$ & $2.48 \pm 1.84$ \\
\hline Total crotch length & $12.20 \pm 9.44$ & $12.90 \pm 8.30$ & $9.47 \pm 7.51$ & $11.52 \pm 6.88$ & $12.69 \pm 10.20$ \\
\hline Mean & 5.3342 & 5.5067167 & 5.6738 & 6.4789833 & 5.95345 \\
\hline
\end{tabular}

Table 5: The distribution of errors between the other measurements and the result of model.

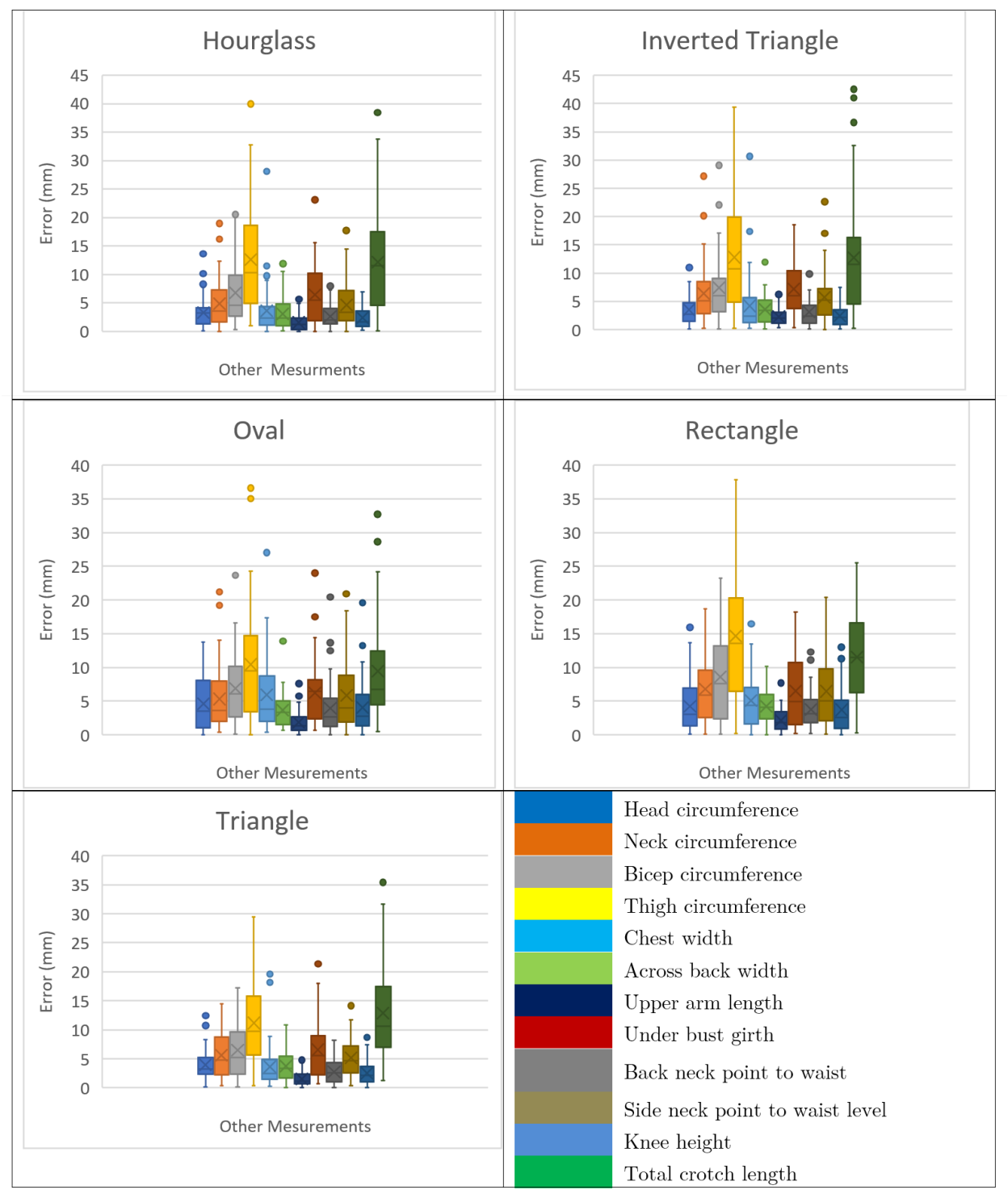




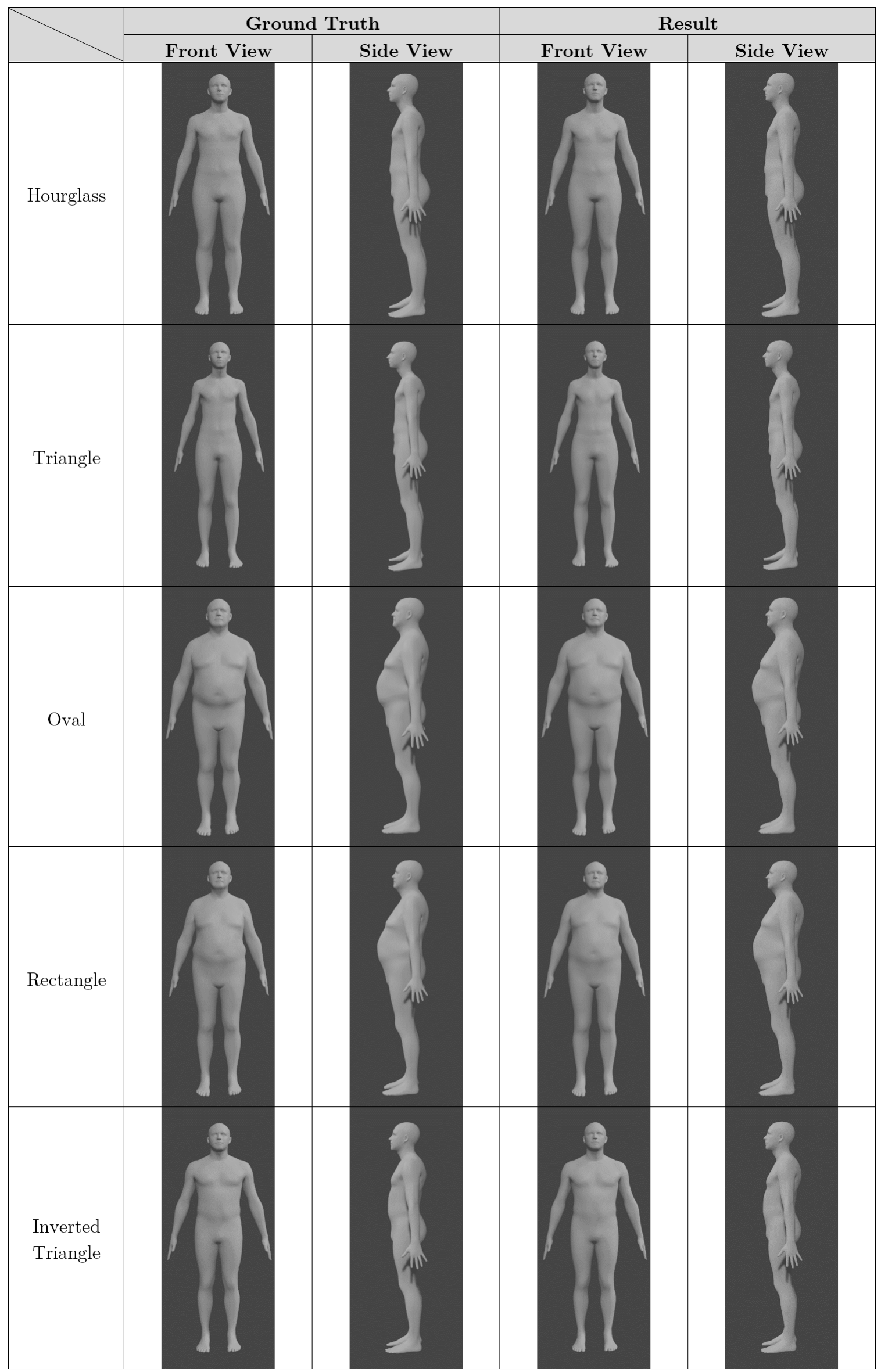

Figure 3: The comparisons between ground truth model and the results in types of body. 


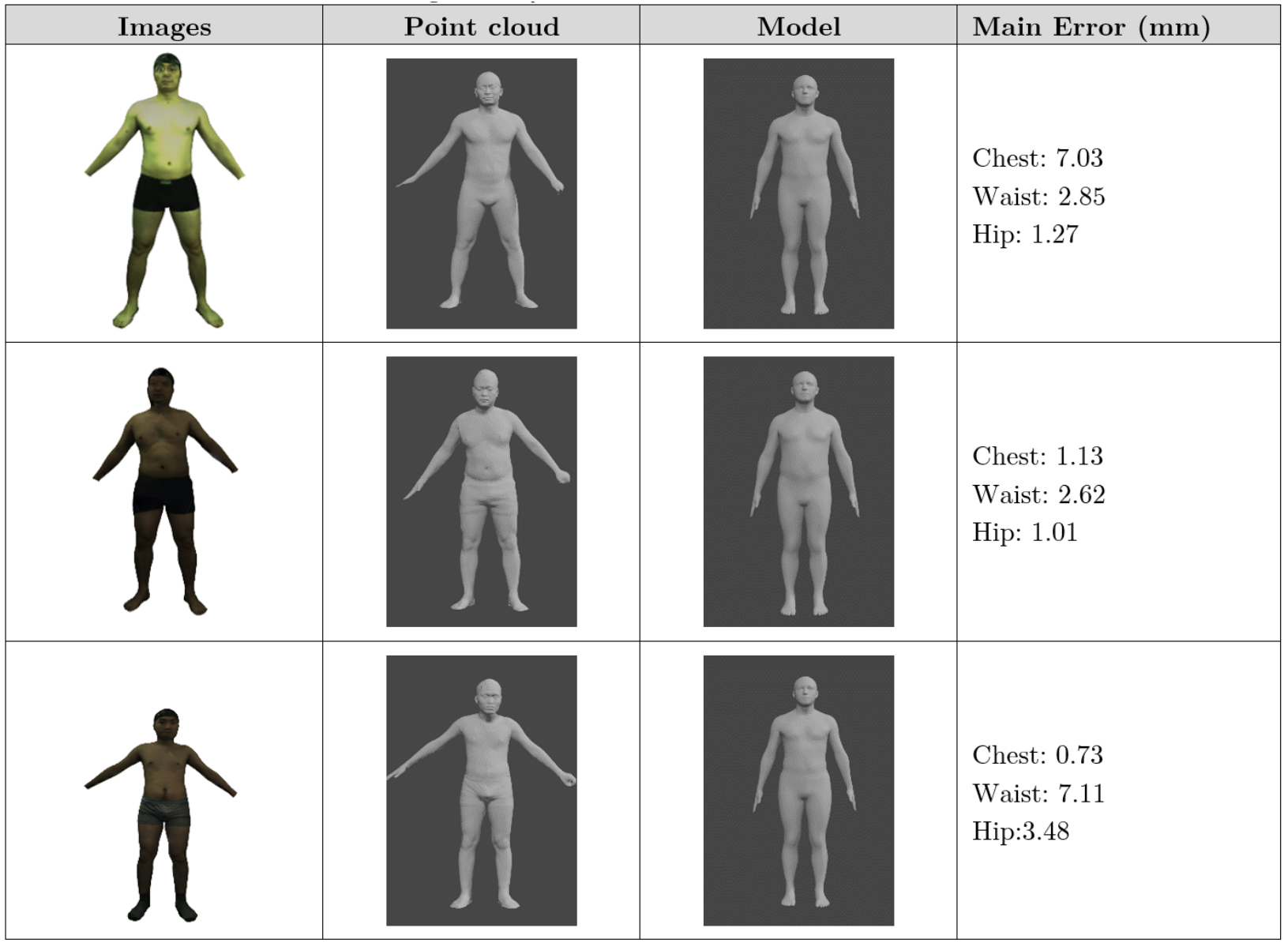

Figure 4: Qualitative results on real data.

is only around $23 \mathrm{~mm}$ in contrast to nearly $40 \mathrm{~mm}$ of the inverted triangle one. The numbers of hourglass, rectangle and triangle types are $33 \mathrm{~mm}, 36 \mathrm{~mm}$ and 32 $\mathrm{mm}$ respectively. However, there are more outliers that appear in oval shape than others. These values could come from special characteristic of this shape which is shown on the next part of this research. On the other hand, the rectangle remains the error stable inside the range of each measurement.

\subsubsection{Qualitative Results}

Fig. 3 below indicates the comparison both in front view and side view between ground truth and 3D model. It is clearly to see that results are acceptable and accurate. In details, thigh circumference of predicted model is slightly smaller than the real data. With oval and rectangle shape, waist is much bigger than other circumferences that causes the effect to other measurements. Hourglass, triangle and inverted triangle body shape have all circumferences and lengths more stable and natural which create more accurate results.

\subsection{Real Dataset on Northern Vietnamese}

The number of real dataset is 75 samples (male), which is collected from two biggest cities in Vietnam: Hanoi and Ho Chi Minh city. The samples are chosen to ensure the diversity of human dataset. Their jobs are included: student, engineer, shipper, worker, famer. The results below describe the effectiveness and the accuracy when applied the algorithm on this dataset. Based on the qualitative results, the differences between images/point cloud dataset and the generated model are small and negligible. Only minor distinctions appear in head and bicep measurements.

The summary on quantitative results below demonstrates the accuracy of proposed algorithm. The mean errors of ten inputs and twelve other measurements are $5.074(\mathrm{~mm})$ and $10.98(\mathrm{~mm})$ respectively. The main errors are head circumference and bicep circumference with $21.51(\mathrm{~mm})$ and $19.32(\mathrm{~mm})$ respectively. These bring the similarity with qualitative results. 
Table 6: Quantitative results on Input Measurements.

\begin{tabular}{|l|c|}
\hline & Error $(\mathrm{mm})$ \\
\hline Overall Height & $3.68 \pm 3.73$ \\
\hline Shoulder breadth & $9.70 \pm 5.14$ \\
\hline Shoulder to crotch & $3.31 \pm 2.60$ \\
\hline Arm length & $4.95 \pm 6.74$ \\
\hline Chest circumference & $4.63 \pm 3.63$ \\
\hline bust point to bust point & $6.38 \pm 3.49$ \\
\hline Waist circumference & $6.03 \pm 5.00$ \\
\hline Upper Hip Circumference & $5.81 \pm 4.29$ \\
\hline Hip circumference & $2.82 \pm 2.03$ \\
\hline Inside leg length & $3.43 \pm 2.34$ \\
\hline Mean & 5.074 \\
\hline
\end{tabular}

Table 7: Quantitative results on others.

\begin{tabular}{|l|r|}
\hline & \multicolumn{1}{|c|}{ Error $(\mathrm{mm})$} \\
\hline Head circumference & $21.51 \pm 12.60$ \\
\hline Neck circumference & $19.81 \pm 9.21$ \\
\hline Bicep circumference & $28.34 \pm 11.13$ \\
\hline Thigh circumference & $7.44 \pm 6.61$ \\
\hline Chest width & $4.60 \pm 3.26$ \\
\hline Across back width & $3.10 \pm 2.25$ \\
\hline Upper arm length & $5.75 \pm 3.71$ \\
\hline Under bust girth & $3.01 \pm 2.53$ \\
\hline Back neck point to waist & $5.46 \pm 4.33$ \\
\hline Side neck point to waist & $3.01 \pm 2.17$ \\
\hline Knee height & $18.51 \pm 13.14$ \\
\hline Total crotch length & 10.98 \\
\hline Mean & \\
\hline
\end{tabular}

\section{Conclusion and Future work}

A novel method for reconstructing 3D model from anthropometric measurements is proposed in this research. Other than previous approaches, we applied a new algorithm (DCGA) to improve the accuracy of reshaping 3D human model from anthropometric measurements. The results in both synthetic data and real data have demonstrated the effectiveness of proposed algorithm. There are two main contributions in this research: (1) applying Diversity Control Oriented Genetic Algorithm to find the best result for $3 \mathrm{D}$ shape model, (2) verifying and validating the results of algorithm on a diverse dataset. In the future, the speed of algorithm would be improved sharply, besides more real dataset should be collected and tested on our algorithm. On the other side, multi-platform user interfaces would be developed and released to research community for many applications.

Acknowledgement: The authors would like to thank all member in 3DR team for their contribution. This research is fully funded by Viettel High Technology Industries Corporation.

\section{References}

[1] Deep, K., And Thakur, M. A new crossover operator for real coded genetic algorithms. Applied Mathematics and Computation 188, 1 (2007), 895911.

[2] Deep, K., And Thakur, M. A new mutation operator for real coded genetic algorithms. Applied Mathematics and Computation 193, 1 (2007), 211230.

[3] Loper, M., Mahmood, N., Romero, J., PonsMoll, G., AND BlaCK, M. J. Smpl: A skinned multi-person linear model. ACM Transactions on Graphics 34, 6 (Oct. 2015).

[4] Pujades, S., Mohler, B., Thaler, A., Tesch, J., Mahmood, N., Hesse, N., Bülthoff, H., AND BLACK, M. J. The virtual caliper: Rapid creation of metrically accurate avatars from $3 \mathrm{~d}$ measurements. IEEE Transactions on Visualization and Computer Graphics 25 (2019), 1887-1897.

[5] Shimodaira, H. A diversity-control-oriented genetic algorithm (dcga): Performance improvement by the reinitialization of the population. GECCO'01, Morgan Kaufmann Publishers Inc., p. $576-583$.

[6] Simmons, K., Istook, C. L., and Devarajan, P. Female figure identification technique (ffit) for appereal part i: Describing female shapes. Journal of textile and apparel, Technology and Management 4, 1 (2004), 1-16.

[7] Streuber, S., Quiros-Ramirez, M. A., Hill, M. Q., Hahn, C. A., Zuffi, S., O'Toole, A., AND BlACK, M. J. Body talk: Crowdshaping realistic 3d avatars with words. ACM Transactions on Graphics 35, 4 (July 2016).

[8] Wuhrer, S., AND Shu, C. Estimating 3d human shapes from measurements. Machine Vision and Applications 24 (2013), 1133-1147.

[9] Zeng, Y., Fu, J., AND ChaO, H. 3d human body reshaping with anthropometric modeling. In Internet Multimedia Computing and Service (Singapore, 2018), B. Huet, L. Nie, and R. Hong, Eds., Springer Singapore, pp. 96-107. 